INCONTINENCE

\section{Bar neurons and urination}

A newly identified subset of excitatory Barrington nucleus (Bar) neurons express oestrogen receptor 1 (ESR1) and control the urethral sphincter in mice. This discovery improves our understanding of the neuronal control of urination.

Immunostaining revealed a small subpopulation of ESR1-expressing Bar (Bar ${ }^{\text {ESR1 }}$ ) neurons in mice distinct from Bar neurons that express corticotropin-releasing hormone (CHC). Fluorescence assays in ESR1-Cre mice using a voluntary urination assay, in which male mice urinate in response to smelling female mouse odour, showed that increases in fluorescence correlated with urination events.

Infection of Bar ${ }^{\text {ESR1 }}$ neurons with an excitatory optogenetic protein, channelrhodopsin 2 (ChR2), showed that, on photostimulation, these neurons caused frequency-dependent urine volume release in freely moving male mice with no female odour stimulation. Photostimulation under anaesthesia caused urine voiding in $46 \%$ of $\mathrm{Bar}^{\mathrm{ESR} 1-\mathrm{ChR} 2}$ mice, but only in $6 \%$ of $\mathrm{Bar}^{\mathrm{CHC}-\mathrm{ChR} 2}$ mice, none of which occurred during the photostimulus window. Photostimulation of filled bladders showed that peak pressure and end pressure were considerably reduced in $\mathrm{Bar}^{\mathrm{ESR} 1-\mathrm{ChR2}}$ mice, owing to abundant liquid release resulting in a sharp decrease in pressure. Photostimultated urine release coincided with a bursting pattern of sphincter activity; urine voiding was pulsatile during the periods. Analysis of data from surgically implanted pressure recorders showed that urethral sphincter bursting patterns corresponded with urination behaviour in response to female odour.

Conversely, infection of Bar neurons with an inhibitory chemogenetic receptor and treatment with its ligand caused reduced urination in response to female odour in $B a r^{E S R 1}$ mice. Infection of Bar ${ }^{E S R 1}$ neurons with an optogenetic inhibitor reduced urination in response to female odour and stopped bursting activity during photoinhibition.

These results show that $\mathrm{Bar}^{\mathrm{ESR} 1}$ neurons are essential for voluntary urinary and sphincter activity in mice. Translating these findings to humans could improve our understanding of voiding dysfunction.

\title{
Targeting TFE3-fusion translocation RCC
}

The creation of a new patient-derived xenograft (PDX) model of TFE3-fusion translocation renal cell carcinoma (tRCC) has enabled the identification of dysregulated PI3K-AKT-mTOR signalling in this disease. Dual PI3K-mTOR inhibition had antitumour activity in vitro and in vivo, providing a novel line of enquiry for drug development for this difficult-to-treat malignancy.

Samples from a lymph node metastasis of micropthalmis transcription factor family tRCC were cultured in mice and then harvested to establish a cell line and for histological and molecular characterization. The resulting RP-R07 cells and PDXs retained the histological features of the original metastasis and had the same strong TFE3 nuclear immunoreactivity. RNA sequencing identified a fusion transcript spanning SFPQ and TFE3.

Analysis of the molecular pathways targeted by the SFPQ-TFE3 fusion gene showed that the PI3K-AKT-mTOR axis was consistently influenced, and many genes related to this pathway were identified as targets of the SFPQ-TFE3 fusion. Disproportionate upregulation of the PI3KAKT-mTOR pathway was observed in three tRCC cell lines.

In vitro, testing of three vertical inhibition schemas to target the PI3K-AKT-mTOR axis showed that simultaneous inhibition of PI3K and mTOR with a dual inhibitor had more antiproliferative activity than PI3K-AKT or mTOR inhibition alone in tRCC. Silencing of TFE3 using small interfering RNA inhibited RP-R07 cell proliferation.

In vivo, treatment with a dual PI3K and mTOR inhibitor resulted in significantly lower RP-R07 PDX tumour weight than control treatment.

Overall, these data suggest that targeting the PI3K-AKT-mTOR axis has therapeutic potential in TFE3-fusion tRCC.

Louise Stone

ORIGINAL ARTICLE Damayanti, N. P. et al. Therapeutic targeting of TFE3/IRS-1/PI3K/mTOR axis in translocation renal cell carcinoma. Clin. Cancer Res. https://doi.org/10.1158/ 1078-0432.CCR-18-0269 (2018)

\section{PROSTATE CANCER}

\section{Novel nucleoporin drives aggressiveness}

A new study has identified a novel mechanistic role for nucleoporin POM121 in prostate cancer progression.

Initial transcriptomic profiling of nuclear pore complex composition in clinical tumour samples identified a subset of nucleoporins that are deregulated during primary-to-metastatic disease progression. A subsequent genetic loss-of-function screen implicated POM121 - the top upregulated nucleoporin - as a key mediator of aggressive tumour biology in vitro and in vivo.

Immunoprecipitation and knockdown assays showed that POM121 exerts its oncogenic effects by enhancing nuclear import through direct interaction with importin- $\beta$, a nuclear import machinery component. Transcriptomic profiling of aggressive prostate cancer cell lines and clinical specimens revealed a common POM121-associated target gene signature related to those of transcription factors MYC and E2F1. Further mechanistic characterization showed that POM121 controls importin$\beta$-dependent nuclear shuttling and signalling of the transcription factors MYC, E2F1, and GATA2, and the androgen receptor (AR).

Treatment of aggressive prostate cancer cells with the importin- $\beta$ inhibitor importazole decreased tumorigenicity, proliferation, therapeutic resistance, and MYC, E2F1, GATA2, and AR nuclear localization. Consistently, importazole (alone or with chemotherapy) improved overall survival and reduced tumour burden and Ki67 expression in xenograft mouse models.

Overall, POM121 enhances prostate cancer aggressiveness by regulating the nuclear localization and activity of key transcription factors, a mechanism that could be therapeutically targeted by disrupting the nuclear import machinery. "In future studies, we will try to identify the tumours that might benefit the most from this strategy, such as those driven by specific transcription factors," concludes author Josep Domingo-Domenech.

Conor A. Bradley

ORIGINAL ARTICLE Rodriguez-Bravo, V. et al. Nuclear pores promote lethal prostate cancer by increasing POM121-driven E2F1, MYC, and AR nuclear import. Cell 174, 1200-1215 (2018) 